Proceedings of the European Conference Physics of Magnetism 2011 (PM'11), Poznań, June 27-July 1, 2011

\title{
Electrical Resistivity and Electronic Structure of the $\mathrm{Tb}_{x} \mathrm{Gd}_{1-x} \mathrm{Ni}_{3}$ System
}

\author{
G. Chękowska*, A. Bajorek, A. Chrobak and M. Kwiecień-Grudziecka
}

August Chełkowski Institute of Physics, University of Silesia, Uniwersytecka 4, 40-007 Katowice, Poland

\begin{abstract}
In the paper the electric properties and electronic structure of the intermetallic $\mathrm{Tb}_{x} \mathrm{Gd}_{1-x} \mathrm{Ni}_{3}$ compounds are presented. The partial replacement of $\mathrm{Gd}$ by $\mathrm{Tb}$ atoms causes the decrease of the Curie temperature $\left(T_{\mathrm{C}}\right)$ and the increase of the residual resistivity. According to the Matthiessen rule the scattering mechanisms in $\rho(T)$ have been analyzed. Moreover, the reduced form of the electrical resistivity $\rho_{Z}\left(T-T_{0}\right)$ indicates a deviation from the linearity for $x>0.2$. This kind of behaviour can be attributed to density of $d$ states near by the Fermi level $\left(E_{\mathrm{F}}\right)$ which are dominated by Ni $3 d$ states. The valence band spectra as well as the core level lines have been analyzed as the influence of $\mathrm{Tb} / \mathrm{Gd}$ substitution on the electronic structure.
\end{abstract}

PACS: 71.20.Eh, 71.20.Lp, 72.15.-v, 72.10.Di, 79.60.-i

\section{Introduction}

The $\mathrm{RT}_{3}$ compounds where $\mathrm{R}$ is rare earth and $\mathrm{T}$ is a transition metal show interesting properties due to the combination of $3 d$ itinerant and $4 f$ localized magnetism [1-17]. One of the first studied group is $\mathrm{RNi}_{3}$. Obviously, its properties depends on the type of $\mathrm{R}$ atoms. In the $\mathrm{GdNi}_{3}$ compound the Curie temperature $T_{\mathrm{C}}=115 \mathrm{~K}$ and the saturation magnetic moment equals $6.55 \mu_{\mathrm{B}} / \mathrm{f} . \mathrm{u}$. [1, 4 , 12, 13]. The $\mathrm{TbNi}_{3}$ compound exhibits non-collinear magnetic structure where the terbium atoms in the same crystallographic position are arranged ferromagnetically but they are turned between two different positions [16]. Thus, the magnetic susceptibility exhibits a large magnetic anisotropy with two different paramagnetic Curie temperatures $\theta_{\|}=9 \mathrm{~K}$ and $\theta_{\perp}=63 \mathrm{~K}$. The saturation magnetic moment equals $7.52 \mu_{\mathrm{B}} /$ f.u. The electrical resistivity exhibits two magnetic transitions, one at about $55 \mathrm{~K}$ (helimagnetic structure of terbium moments along $c$ axis) and the second at about $80 \mathrm{~K}$ (transition from ferromagnetic to paramagnetic state) [16].

In this paper we are focused on the influence of terbium substitution on the electric properties and the electronic structure in the $\mathrm{Tb}_{x} \mathrm{Gd}_{1-x} \mathrm{Ni}_{3}$ series.

\section{Experimental details}

The polycrystalline samples $\mathrm{Tb}_{x} \mathrm{Gd}_{1-x} \mathrm{Ni}_{3}(x=0.0$, $0.2,0.5,0.8,1.0)$ were prepared by arc-melting from high purity elements under argon atmosphere. All samples were wrapped in tantalum foil, placed in quartz tubes and annealed at $900^{\circ} \mathrm{C}$ for one week. The crystal structure was checked by means of X-ray diffraction (XRD) using Siemens D5000 diffractometer. The electrical resistivity $\rho(T)$ was measured quasi-continuously at a slowly

\footnotetext{
* corresponding author; e-mail: grazyna.chelkowska@us.edu.pl
}

changing temperature $(4.2-300 \mathrm{~K})$ by a standard four-probe technique. The X-ray photoelectron spectroscopy (XPS) measurements were performed with the use of PHI 5700/660 Physical Electronics spectrometer. The spectra were analyzed at room temperature using monochromatized $\mathrm{Al} K_{\alpha}$ radiation $(1486.6 \mathrm{eV})$. The samples were fractured and measured in vacuum of $10^{-10}$ Torr.

\section{Results and discussion}

\subsection{Electrical resistivity}

The $\rho(T)$ dependence for the $\mathrm{Gd}_{1-x} \mathrm{~Tb}_{x} \mathrm{Ni}_{3}$ systems is presented in Fig. 1a. The $\mathrm{Tb} / \mathrm{Gd}$ substitution causes the decrease of the temperature of the magnetic phase transition $\left(T_{\mathrm{C}}\right)$. Similar behaviour has been observed from magnetic measurements [18]. According to the Matthiessen rule the electrical resistivity can be described as

$$
\rho(T)=\rho_{0}+\rho_{\mathrm{ph}}(T)+\rho_{\mathrm{mag}}(T),
$$

where $\rho_{0}$ - residual resistivity, $\rho_{\mathrm{ph}}$ - phonon contribution, $\rho_{\text {mag }}-$ magnetic part. The $\rho_{\mathrm{ph}}$ for pure metals in the high temperature range follows the Bloch-Grüneisen dependence and changes linearly with $T$. The $\rho_{\text {mag }}$ represents a spin-disorder contribution which is caused by the scattering of the conduction electrons on $4 f$ moments and contribution correlated with spin fluctuations. In our case the value of $\rho_{0}$ increases with the terbium substitution (see Table). It may be a result of crystal disorder which appears in the crystal lattice during substitution.

Analyzing the $\rho(T)$ curve below $T_{\mathrm{C}}$ we have to take into account the phonon contribution $\left(\rho_{\mathrm{ph}} \sim T^{5}\right)$ and the scattering of $s$ electrons to free $d$ states $\left(\rho_{s d} \sim T^{3}\right)$. Therefore, the following expression has been used:

$$
y=a x^{5}-b x^{3}+c x^{n} .
$$

For the compounds with $x \leq 0.2$ the $n$ is close to 2 which indicates the scattering on the spin waves (magnons). In the case of compounds with $x>0.2$ the resistivity below 


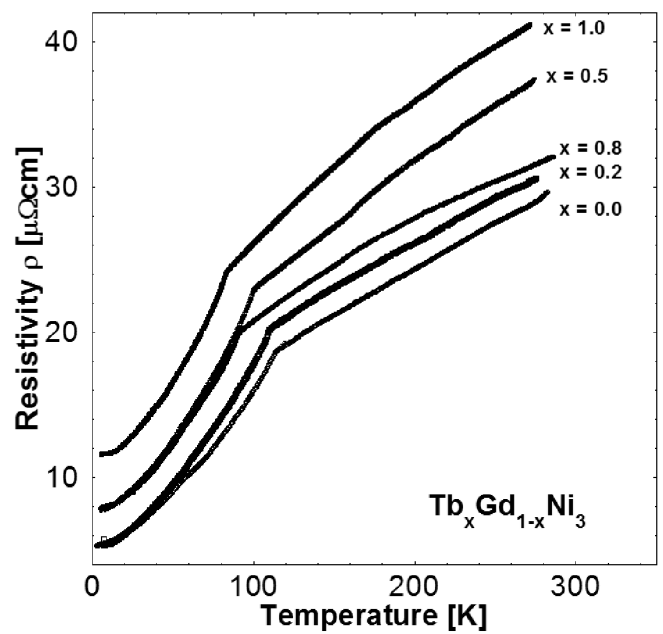

Fig. 1. Temperature dependence of the electrical resistivity for the $\mathrm{Tb}_{x} \mathrm{Gd}_{1-x} \mathrm{Ni}_{3}$ system.
$T_{\mathrm{C}}$ can be rather fitted by using the equation

$$
y=a x^{5}-b x^{3}+c x^{2} \exp (-d / x),
$$

where $a, b, c$ are constants and parameter $d$ is proportional to the energy gap $\left(d=\Delta / 2 k_{\mathrm{B}}\right)$. The exponential contribution may be the result of a magnetic anisotropy which was already observed by Hashimoto et al. in the $\mathrm{TbNi}_{3}$ compound [16].

The analysis of parameters obtained from the fitting of $\rho(T)$ dependence indicates that all of them increase with $\mathrm{Gd} / \mathrm{Tb}$ substitution. At the same time the value of energy gap $\Delta$ in the spin waves spectra also increases (see Table). This change is caused by using additional energy which is needed to reverse the localized spins opposite to the anisotropy field.

The reduced form of the electrical resistivity can be represented as

$$
\rho_{Z}=\rho(T)-\rho\left(T_{0}\right) / \rho\left(T_{1}\right)-\rho\left(T_{0}\right),
$$

in the assumption where $T_{0}=140 \mathrm{~K}$ and $T_{1}=260 \mathrm{~K}$.

TABLE

\begin{tabular}{|c|c|c|c|c|c|c|c|}
\hline \multirow[t]{2}{*}{$x$} & \multirow[t]{2}{*}{$T_{\mathrm{C}}[\mathrm{K}]$} & \multirow[t]{2}{*}{$\rho_{0}[\mu \Omega \mathrm{cm}]$} & $a \times 10^{-10}$ & $b \times 10^{-5}$ & $c \times 10^{-3}$ & \multirow{2}{*}{\multicolumn{2}{|c|}{$n$}} \\
\hline & & & \multicolumn{3}{|c|}{$\left[\mu \Omega \mathrm{cm} \mathrm{K}^{-5}\right]$} & & \\
\hline \multirow{5}{*}{0.0} & \multirow{5}{*}{115} & \multirow{5}{*}{5.17} & \multicolumn{5}{|c|}{$55-113 \mathrm{~K}$} \\
\hline & & & 3.68 & 1.33 & 1.45 & \multicolumn{2}{|c|}{2.08} \\
\hline & & & \multicolumn{5}{|c|}{$4.2-50 \mathrm{~K}$} \\
\hline & & & 4.19 & 1.97 & 3 & \multicolumn{2}{|c|}{1.90} \\
\hline & & & \multicolumn{5}{|c|}{$T<T_{\mathrm{C}}$} \\
\hline 0.2 & 110 & 5.29 & 3.69 & 1.51 & 2.73 & \multicolumn{2}{|c|}{1.97} \\
\hline & & & & & & $d[\mathrm{~K}]$ & $\Delta[\mathrm{meV}]$ \\
\hline 0.5 & 101 & 7.75 & 6.02 & 2.04 & 3.02 & 2.89 & 0.25 \\
\hline 0.8 & 90 & 7.79 & 8.50 & 2.64 & 3.50 & 8.22 & 0.71 \\
\hline 1.0 & 83 & 11.45 & 16.2 & 3.63 & 4.31 & 8.44 & 0.73 \\
\hline
\end{tabular}

The transport properties of the $\mathrm{Tb}_{x} \mathrm{Gd}_{1-x} \mathrm{Ni}_{3}$ compounds.

The $\rho_{Z}\left(T-T_{0}\right)$ concerns paramagnetic range and represents the phonon contribution and the $s-d$ scattering on the non-ordered spins. Thus, $\rho_{Z}$ exhibits linear behaviour above $T_{\mathrm{C}}$ only for the samples with $x \leq 0.2$. In the case of higher $\mathrm{Tb}$ content the $\rho_{Z}\left(T-T_{0}\right)$ resistivity deviates from the linearity and shows a positive curvature. This kind of behaviour can be explained as the strong dependence on density of $d$ states nearby the Fermi level.

\subsection{Electronic structure}

For all studied samples valence bands (VB) spectra nearby the Fermi level $\left(E_{\mathrm{F}}\right)$ are dominated by the Ni $3 d$ states (Fig. 2a). The terbium substitution causes the decrease of the intensity of $\mathrm{Gd} 4 f$ line which is located at $8 \mathrm{eV}$. Simultaneously, the multiplet structure of $\mathrm{Tb}$ appears. This structure consists of several lines located at $2 \mathrm{eV}, 7.4 \mathrm{eV}, 9.1 \mathrm{eV}$ and $10.2 \mathrm{eV}$. The broadening of states nearby to $E_{\mathrm{F}}$ is particularly visible for $x=0.8$ and 1.0 terbium concentration. It is the result of $\mathrm{Tb}$ substitution and the increase of the intensity of $\mathrm{Tb} 4 f$ states (Fig. 2a and b). For the compounds with $x \geq 0.5$ the slight increase of intensity of states on the $E_{\mathrm{F}}$ is visible. This behaviour could be the result of $f-d$ interaction. Simultaneously in the resistivity measurements it has been shown that for these compounds the resistivity increases with the temperature faster than for other compounds $(x \leq 0.2)$. On the one hand, it may be the effect of decrease of $5 d$ electrons number (terbium has two $4 f$ electron more but one none $5 d$ electron than gadolinium). On the other hand, it may be the result of the decrease of relaxation time between collisions as a consequence of higher probability of electrons scattering into free $d$ states. 

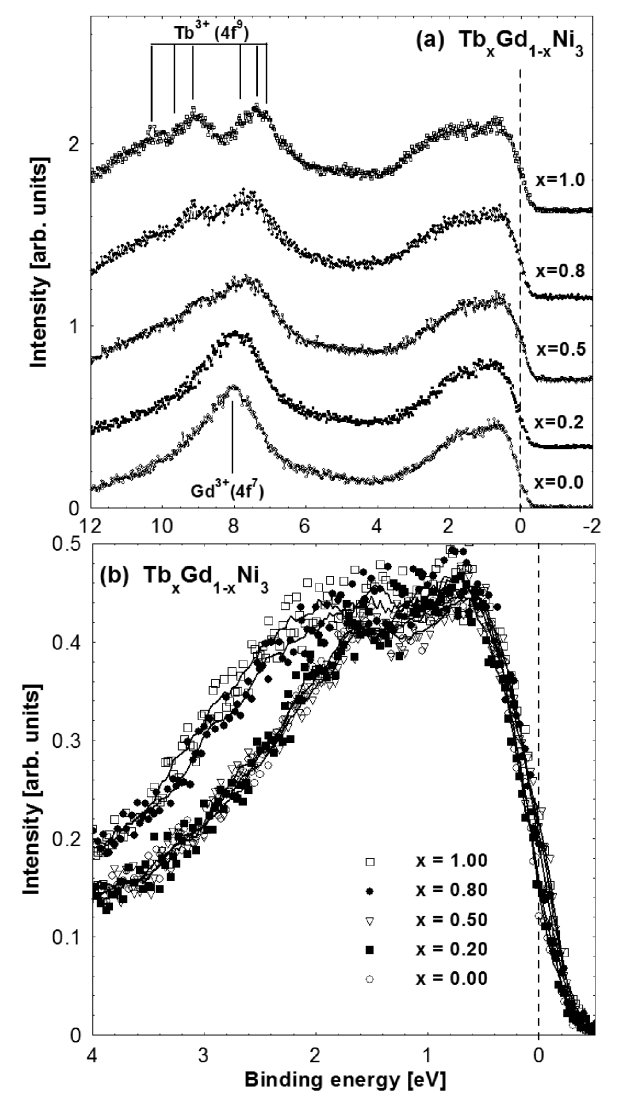

Fig. 2. (a) Valence band spectra in the broad binding energy range and (b) near by the Fermi level for the $\mathrm{Tb}_{x} \mathrm{Gd}_{1-x} \mathrm{Ni}_{3}$ series where $x=0.0,0.2,0.5,0.8$, and 1.0 .

\section{Summary}

The aim of presented work was to study the electric properties as well as the electronic structure of the $\mathrm{Tb}_{x} \mathrm{Gd}_{1-x} \mathrm{Ni}_{3}$ series. All results can be concluded as follows:

- The value of the temperature of phase transition $T_{\mathrm{C}}$ obtained from $\rho(T)$ decreases with $\mathrm{Gd} / \mathrm{Tb}$ substitution. The fitting of $\rho(T)$ dependence for $x \leq 0.2$ below $T_{\mathrm{C}}$ indicates the scattering on the spin waves whereas for $x>0.2$ is rather connected with a magnetic anisotropy. Additionally, the reduced resistivity $\rho_{Z}\left(T-T_{0}\right)$ exhibits linear dependence for $x \leq 0.2$ samples and for higher $\mathrm{Tb}$ content deviates from the linearity. This deviation can be connected with the change of the electronic structure.

- The VB spectra nearby $E_{\mathrm{F}}$ are dominated by the $\mathrm{Ni} 3 d$ states. For the compounds with $x \geq 0.5$ the slight increase of intensity of states on the $E_{\mathrm{F}}$ has been observed. Simultaneously, the $\rho_{Z}\left(T-T_{0}\right)$ indicates the deviation from the linearity. It may be the effect of decrease of $5 d$ electron number but also the result of the decrease of relaxation time between collisions as a consequence of higher probability of electrons scattering into free $d$ states.

\section{Acknowledgments}

This work was partially supported by Ministry of Science and Higher Education, grant no. N N202 200039.

\section{References}

[1] K.H.J. Buschow, Rep. Prog. Phys. 40, 1179 (1977).

[2] J. Yakinthos, D. Paccard, Solid State Commun. 10, 989 (1972).

[3] J. Rossat-Mignod, J. Yakinthos, Phys. Status Solidi B 47, 239 (1971).

[4] D. Paccard, R. Pauthenet, Compt. Rend. Acad. Sci. Paris 264, 1056 (1967).

[5] E. Burzo, J. Laforest, Compt. Rend. Acad. Sci. Paris 274, 114 (1972).

[6] J.E. Greedan, in: Proc. 9th Rare Earth Res. Conf., Virginia Polytechnic Inst. and State Univ., Blacksburg (Va) 1971, p. 208.

[7] E. Burzo, D. Seitabla, Solid State Commun. 37, 663 (1981).

[8] F. Porarian, T. Tajabor, Phys. Status Solidi A 61, 537 (1980).

[9] S.K. Arif, D.S.P. Bunbury, Phys. Status Solidi A 33, 91 (1976).

[10] S.C. Tsai, K.S.V.L. Narasimhan, J. Appl. Phys. 45, 3582 (1974).

[11] K.S.V.L. Narasimhan, R.A. Butera, R.S. Craig, J. Appl. Phys. 44, 879 (1973).

[12] A. Chrobak, A. Bajorek, G. Chełkowska, G. Haneczok, M. Kwiecień, Phys. Status Solidi A 206, 731 (2009).

[13] A. Bajorek, A. Chrobak, G. Chełkowska, M. Kwiecień, J. Alloys Comp. 485, 6 (2009).

[14] E. Burzo, D. Seitabla, J. Phys. F, Met. Phys. 11, L255 (1981).

[15] E. Burzo, D. Seitabla, J. Phys. F, Met. Phys. 12, 2675 (1982).

[16] Y. Hashimoto, H. Fuji, T. Okamoto, Y. Makihara, J. Magn. Magn. Mater. 70, 291 (1987).

[17] P. de V. du Plessis, T. Germishuyse, J. Magn. Magn. Mater. 104-107, 1349 (1992).

[18] A. Bajorek, G. Chełkowska, A. Chrobak, M. Kwiecień-Grudziecka, Solid State Phenom. 170, 109 (2011). 